\title{
Synthesis and Biological Evaluation of (E)-N'- Benzylidene-7-methyl-2-propyl-1H-benzo[d] imidazole-5-carbohydrazides as Antioxidant, Anti-inflammatory and Analgesic agents
}

https://doi.org/10.1515/hc-2019-0009

Received November 12, 2018; accepted January 11, 2019.

\begin{abstract}
E)- $N$ '-Benzylidene-7-methyl-2-propyl-1H-benzo [d]imidazole-5-carbohydrazides (5a-r) have been synthesized from 7-methyl-2-propyl-1H-benzo[ $d]$ imidazole-5-carbohydrazide (3) by condensing with different aromatic aldehydes (4a-r). Title compounds (5a-r) were evaluated for in vitro antioxidant activity and based on their potential for antioxidant property, selected compounds 5d and 5m-p were screened for in vivo anti-inflammatory and analgesic activity. The results indicate that the compound $\mathbf{5 0}$ and $\mathbf{5 p}$ are effective against anti-inflammatory and analgesic activity. The biological data was further supported by molecular docking studies, which revealed the binding pattern and the affinity of the molecules in the active site of COX-2.
\end{abstract}

Keywords: Benzimidazolyl hydrazones, antioxidant activity, anti-inflammatory activity, analgesic activity and acid hydrazide

\section{Introduction}

Non-steroidal anti-inflammatory drugs (NSAIDs) are considered as one of the most widely used therapeutics to

* Corresponding author: Ramu Kakkerla, Department of Chemistry, Satavahana University, Karimnagar 505001, Telangana State, India Ramamurthy Katikireddy, Department of Chemistry, JNTU, Kakinada 533003, Andhra Pradesh, India, Enantilabs Pvt. Ltd., JN Pharmacity, Visakapatnam 531019, Andhra Pradesh, India M. P. S. Murali Krishna, Department of Chemistry, Andhra Polytechnic College, Kakinada 533003, Andhra Pradesh, India Gandamalla Durgaiah and Y. N. Reddy, Department of Pharmacology and Toxicology, Kakatiya University, Warangal 506009, Telangana State, India

*Mavurapu Satyanarayana, Department of Pharmaceutical Chemistry, Telangana University, Nizamabad 503322, Telangana State, India alleviate pain and inflammation, especially arthritis. Most of the currently available NSAIDs have limitations for therapeutic uses, as they cause gastrointestinal and other side effects which are inseparable from their pharmacological activities. This prompts us for identification of lead structures that may be useful in designing new, potent drugs with less toxic effects $[1,2]$.

5-Substituted benzimidazoles are privileged heterocycles for medicinal chemists due to their broad range of biological activities such as anti-inflammatory [3], anticancer [4] and more specifically benimidazole-5-carboxylic acid and its derivatives are promising agents for hepatitis C virus infections [5, 6, 7], Glycoprotein IIb/ IIIa inhibitors [8], Glutaminyl Cyclase inhibitors [9], and checkpoint kinase inhibitor [10] activities. Further the hydrazones plays a key role in medicinal chemistry due to the azomethine (-NH-N=CH-) connected with carbonyl group, and is responsible for different pharmaceutical applications, such as anti-inflammatory [11, 12], anticancer [13], antiviral [14], anticonvulsant [15], antiprotozoal [16], antimalerial [17], antileshmenial [18], antiamoebic [19], antimicrobial [20], and analgesic [21] activity. Based on the pharmacological activities of both the benzimidazoles and hydrazones, and our quest in search of new biologically active molecules [22-24], herein, we report the design, synthesis, antioxidant, anti-inflammatory and analgesic activity of (E)- $N$ '-Benzylidene-7-methyl-2-propyl$1 H$-benzo[d]imidazole-5-carbohydrazides (5a-r).

\section{Results and discussion}

\section{Chemistry}

The synthesis of target molecules was achieved as outlined in scheme-1. The reaction sequences for the synthesis 
of target hydrazones (5a-r), starts from methyl 4-butyriamido-3-methyl-5-nitrobenzoate (1) [25], which underwent reductive cyclisation to yield methyl 7-methyl-2-n-propyl-1 $H$-benzo[d]imidazole-5-carboxylate (2) [26]. In the process, compound- 1 was reduced with $\mathrm{Na}_{2} \mathrm{~S}_{2} \mathrm{O}_{4}$ in water to afford methyl 3-amino-4-butyriamido-4-methylbenzoate, which in turn internally cyclised by nucleophilic addition of amino group to the adjacent amide carbonyl to form compound-2. The resulting methyl ester (2) was treated with hydrazine hydrate to afford acid hydrazide (3), which was confirmed by the stretching frequencies of $\mathrm{NH}$ and $\mathrm{NH}_{2}$ in the IR spectrum at 3463, 3319, 3292 $\mathrm{cm}^{-1}{ }^{1}{ }^{1} \mathrm{H}$ NMR spectrum of (3) displayed two singlets at $\delta$ 4.49 and $\delta 9.78$ ppm corresponding to $\mathrm{NH}_{2}$ and $\mathrm{NH}$ protons of acid hydrazide respectively. The title compounds (5a-r) were prepared by condensing the acid hydrazide with various aromatic aldehydes (4a-r) in methanol in the presence of catalytic amount of glacial acetic acid under reflux conditions. The target hydrazones (5a-r) were confirmed by IR, ${ }^{1} \mathrm{H}$ NMR, ${ }^{13} \mathrm{C}$ NMR and Mass spectral data. In the ${ }^{1} \mathrm{H}$ NMR spectrum of $\mathbf{5 a}$, absence of $\mathrm{NH}_{2}$ signal at $\delta 4.49$ ppm and appearance of new singlet due to azomethine proton (- $\mathrm{CH}=\mathrm{N}-)$ at $\delta 8.51 \mathrm{ppm}$ confirms the structure of $\mathbf{5 a}$. Further the molecular ion $[\mathrm{M}+\mathrm{H}]^{+}$at $\mathrm{m} / z 321$ in the mass spectrum of $\mathbf{5 a}$ confirms its structure.

Reagents and conditions: (i) $\mathrm{Na}_{2} \mathrm{~S}_{2} \mathrm{O}_{4}, \mathrm{H}_{2} \mathrm{O}$, reflux, $4 \mathrm{~h}, 86 \%$ (ii) $\mathrm{N}_{2} \mathrm{H}_{4}-\mathrm{H}_{2} \mathrm{O}$, ethanol, reflux, $10 \mathrm{~h}, 90 \%$ (iii) Ar-CHO (4a-r), gla.AcOH, $\mathrm{MeOH}$, reflux, 4-6 h, $88-92 \%$

\section{Antioxidant Activity}

The benzimidazolyl hydrazones (5a-r) have been screened for their in vitro antioxidant activity. The results indicates that the compound $\mathbf{5 n}$ has shown potent antioxidant activity with $\mathrm{IC}_{50}$ value of $9.40 \pm 1.04 \mu \mathrm{g} / \mathrm{mL}$, followed by compounds $\mathbf{5 0}, \mathbf{5 m}, \mathbf{5 p}$ and $\mathbf{5 d}$ with the $\mathrm{IC}_{50}$ values of $12.39 \pm 1.26,13.60 \pm 1.37,16.27 \pm 1.37$ and $18.31 \pm 1.38$ $\mu \mathrm{g} / \mathrm{mL}$ respectively. Rest of the compounds $\mathbf{5 a - c ,} \mathbf{5 e}, \mathbf{5} \mathbf{f}-\mathbf{l}$, $\mathbf{5 q}$ and $\mathbf{5 r}$ have shown moderate to good antioxidant activity (Table 1 ). The selected compounds $\mathbf{5 d}$ and $\mathbf{5} \mathbf{m}$-p were further screened for their anti-inflammatory and analgesic activity.

\section{Anti-inflammatory activity}

The title compounds $\mathbf{5 d}$ and $\mathbf{5 m}$-p were screened for their anti-inflammatory and analgesic activity based on their potential for antioxidant property. The tested compounds showed good to excellent anti-inflammatory activity in terms of decreased paw volume as shown in table 2 . The compounds $\mathbf{5 d}$ and $\mathbf{5 m} \mathbf{m}-\mathbf{p}$ exhibited the anti-inflammatory activity in the range of 25.60 to $45.50 \%$ after $1 \mathrm{~h}, 43.15-59.38 \%$ after $2 \mathrm{~h}, 58.30-81.40 \%$ after $4 \mathrm{~h}$ and $64.70-90.30 \%$ after $6 \mathrm{~h}$, where as the $\%$ of inhibition for reference drug (Indomethacin) is $43.30 \%$, $56.80 \%, 74.56 \%$ and $85.90 \%$ at respective time intervals. These results indicate that the selected compounds are showing comparable anti-inflammatory activity with<smiles>CCCC(=O)Nc1c(C)cc(C(=O)OC)cc1[N+](=O)[O-]</smiles><smiles>[3H]/C=N/NC(=O)c1cc(C)c2[nH]c(CCC)nc2c1</smiles><smiles>CCCc1nc2cc(C(=O)NN)cc(C)c2[nH]1</smiles>

5a: $\mathrm{Ar}=\mathrm{C}_{6} \mathrm{H}_{5} ; \mathbf{5 b}: \mathrm{Ar}=4-\mathrm{OCH}_{3} \mathrm{C}_{6} \mathrm{H}_{4} ; \mathbf{5 c}: \mathrm{Ar}=2-\mathrm{OH} \mathrm{C}_{6} \mathrm{H}_{4} ; \mathbf{5 d}: \mathrm{Ar}=3-\mathrm{ClC}_{6} \mathrm{H}_{4} ; \mathbf{5 e :} \mathrm{Ar}=3-\mathrm{BrC}_{6} \mathrm{H}_{4} ; \mathbf{5 f}: \mathrm{Ar}=3-\mathrm{OCH}_{3} \mathrm{C}_{6} \mathrm{H}_{4}$; 5g: $\mathrm{Ar}=3,4-\left(\mathrm{OCH}_{3}\right)_{2} \mathrm{C}_{6} \mathrm{H}_{3} ; \mathbf{5 h}: \mathrm{Ar}=4-\mathrm{CNC}_{6} \mathrm{H}_{4} ; \mathbf{5 i}: \mathrm{Ar}=2$-furyl; $\mathbf{5 j}: \mathrm{Ar}=2$-thienyl; $\mathbf{5 k}: \mathrm{Ar}=1$-napthyl; $\mathbf{5 l}: \mathrm{Ar}=2-\mathrm{CH}_{3} \mathrm{C}_{6} \mathrm{H}_{4}$; $\mathbf{5 m : ~} \mathrm{Ar}=4-\mathrm{NO}_{2} \mathrm{C}_{6} \mathrm{H}_{4} ; \mathbf{5 n}: \mathrm{Ar}=5-\mathrm{Br}, 2-\mathrm{OHC}_{6} \mathrm{H}_{3} ; \mathbf{5 o :} \mathrm{Ar}=2,4-(\mathrm{Cl})_{2}, \mathrm{C}_{6} \mathrm{H}_{3} ; \mathbf{5 p :} \mathrm{Ar}=3,5-(\mathrm{Cl})_{2}, 2-\mathrm{OHC}_{6} \mathrm{H}_{2} ; \mathbf{5 q}: \mathrm{Ar}=4-\mathrm{OH} \mathrm{C}_{6} \mathrm{H}_{4}$; 5r: $\mathrm{Ar}=4-\mathrm{OH}, 3-\mathrm{OCH}_{3} \mathrm{C}_{6} \mathrm{H}_{3}$

Scheme 1 Synthesis of (E)-N'-benzylidene-7-methyl-2-propyl-1H-benzo[d]imidazole-5-carbohydrazides (5a-r). 
the reference standard. Compound $\mathbf{5 o}$ found to be the most effective anti-inflammatory activity with $\%$ of inhibition of 45.50, 59.38, 81.40, and 90.30 at respective time intervals of $1 \mathrm{~h}, 2 \mathrm{~h}, 4 \mathrm{~h}$ and $6 \mathrm{~h}$, followed by compound 5 p with \% of inhibition of 43.30, 57.62, 79.51, and 83.50 (Table 2). The other compounds showed good to moderate anti-inflammatory activity. The results indicate that the presence of electron withdrawing groups like halogens and nitro on phenyl ring is playing a crucial role in the activity.

Table 1 Antioxidant activity of compounds (5a-r)

\begin{tabular}{lr}
\hline Compound & $\mathrm{IC}_{50}(\mu \mathrm{g} / \mathrm{mL})$ \\
\hline $\mathbf{5 a}$ & $49.28 \pm 3.03$ \\
$\mathbf{5 b}$ & $32.17 \pm 2.87$ \\
$\mathbf{5 c}$ & $29.10 \pm 1.60$ \\
$\mathbf{5 d}$ & $18.31 \pm 1.38$ \\
$\mathbf{5 e}$ & $26.81 \pm 2.10$ \\
$\mathbf{5 f}$ & $29.96 \pm 2.81$ \\
$\mathbf{5 g}$ & $24.79 \pm 3.03$ \\
$\mathbf{5 h}$ & $30.83 \pm 2.93$ \\
$\mathbf{5 i}$ & $23.19 \pm 1.72$ \\
$\mathbf{5 j}$ & $30.08 \pm 2.60$ \\
$\mathbf{5 k}$ & $20.05 \pm 1.27$ \\
$\mathbf{5 l}$ & $25.97 \pm 2.18$ \\
$\mathbf{5 m}$ & $13.60 \pm 1.37$ \\
$\mathbf{5 n}$ & $9.40 \pm 1.04$ \\
$\mathbf{5 0}$ & $12.39 \pm 1.26$ \\
$\mathbf{5 p}$ & $16.27 \pm 1.39$ \\
$\mathbf{5 q}$ & $24.70 \pm 2.29$ \\
$\mathbf{5 r}$ & $38.28 \pm 3.07$ \\
Std. & $7.50 \pm 0.89$ \\
(Ascorbic acid) & \\
\hline
\end{tabular}

\section{Analgesic activity}

Analgesic activity of test compounds $\mathbf{5 d}$ and $\mathbf{5 m}$-p were evaluated and the results are presented in table 3. Compound 50 exhibited excellent analgesic activity about $36.50 \%, 53.40 \%, 60.85 \%, 78.38$ and $94.20 \%$ at respective time intervals of $30 \mathrm{~min}, 60 \mathrm{~min}, 90 \mathrm{~min}, 120 \mathrm{~min}$ and $180 \mathrm{~min}$, which is comparable with the activity of standard drug Diclofenac sodium. Rest of the compounds $\mathbf{5 d}, \mathbf{5 m}, \mathbf{5 n}$ and $\mathbf{5 p}$ showed moderate analgesic activity. The difference in the activity may be attributable to the diverse structural variations of the synthesized compounds.

\section{Molecular docking studies}

The crystal structure of Cyclooxygenase-2 (COX-2, prostaglandin synthase-2) from mouse has been selected for molecular docking [27] and carried out the docking studies of $\mathbf{5 d}, \mathbf{5 m - 5 p}$ and the reference molecule indomethacin with COX-2 protein. The indomethacin was also used as reference standard in the in vivo experimental analysis of anti-inflammatory activity of the synthesised molecules and hence selected the COX-2 protein with indomethacin for protein ligand binding analysis in the present study. All the selected five molecules have same core and differentiate at the phenyl substitutions showed similar binding conformations toward COX-2 protein. The crystal structure of PDB_ID:4COX contains a "heme" and indomethacin molecule in the active site. To validate the docking software, we have docked the indomethacin in to the same binding pocket of COX-2, and obtained the same result with similar orientation and minimal root mean square deviation (RMSD). The binding structure

Table 2 Anti-inflammatory activity of synthesized compounds $5 \mathrm{~d}$ and $\mathbf{5 m - p}$

\begin{tabular}{|c|c|c|c|c|c|c|c|c|c|}
\hline \multirow{2}{*}{ Test Samples } & \multirow{2}{*}{$0 \mathrm{hr}$} & \multirow{2}{*}{$1 \mathrm{hr}$} & \multirow{2}{*}{$2 \mathrm{hr}$} & \multirow{2}{*}{$4 \mathrm{hr}$} & \multirow{2}{*}{$6 \mathrm{hr}$} & \multicolumn{4}{|c|}{$\%$ of Inhibition } \\
\hline & & & & & & $1 \mathrm{hr}$ & $2 \mathrm{hr}$ & $4 \mathrm{hr}$ & $6 \mathrm{hr}$ \\
\hline Control & $0.43 \pm 0.06$ & $0.47 \pm 0.03$ & $0.9 \pm 0.03$ & $1.02 \pm 0.08$ & $0.89 \pm 0.01$ & - & - & - & - \\
\hline $\begin{array}{l}\text { Std. } \\
\text { (Indomethacin) }\end{array}$ & $0.34 \pm 0.08^{a}$ & $0.52 \pm 0.04^{*}$ & $0.72 \pm 0.04^{\#}$ & $0.63 \pm 0.03^{a}$ & $0.41 \pm 0.028^{a}$ & 43.30 & 56.80 & 74.56 & 85.90 \\
\hline $5 d$ & $0.32 \pm 0.03^{\#}$ & $0.32 \pm 0.04^{\#}$ & $0.53 \pm 0.02^{\#}$ & $0.37 \pm 0.08^{\#}$ & $0.23 \pm 0.1^{\#}$ & 42.58 & 54.73 & 68.82 & 81.90 \\
\hline $5 \mathrm{~m}$ & $0.4 \pm 0.03^{\mathrm{ns}}$ & $0.33 \pm 0.06^{\#}$ & $0.67 \pm 0.08^{a}$ & $0.82 \pm 0.04^{\#}$ & $0.39 \pm 0.04^{\#}$ & 38.20 & 47.80 & 64.72 & 73.28 \\
\hline $5 n$ & $0.45 \pm 0.08^{\mathrm{ns}}$ & $0.41 \pm 0.02^{\mathrm{ns}}$ & $0.38 \pm 0.05^{\#}$ & $0.51 \pm 0.02^{\#}$ & $0.38 \pm 0.08^{\#}$ & 25.60 & 43.15 & 58.30 & 64.70 \\
\hline 50 & $0.25 \pm 0.05^{\#}$ & $0.32 \pm 0.01^{\#}$ & $0.45 \pm 0.02^{\#}$ & $0.67 \pm 0.03^{a}$ & $0.71 \pm 0.03^{\#}$ & 45.50 & 59.38 & 81.40 & 90.30 \\
\hline $5 p$ & $0.35 \pm 0.02^{*}$ & $0.29 \pm 0.02^{\#}$ & $0.73 \pm 0.03^{\#}$ & $0.59 \pm 0.05^{\#}$ & $0.48 \pm 0.07^{\#}$ & 43.30 & 57.62 & 79.51 & 83.50 \\
\hline
\end{tabular}

(Data are Mean $\pm S D ;{ }^{*} p<0.05,{ }^{a} p<0.01$, ${ }^{*} p<0.001$ vs Control), ${ }^{\text {ns }} p>0.05$ followed by Dunnet’s test.

All other compounds, except $5 \mathrm{~m}$ and $\mathbf{5 n}$ showed the significant activity and comparison with the standard drug indomethcin. 
Table 3 Analgesic activity of synthesized compounds $5 \mathrm{~d}$ and $5 \mathrm{~m}-\mathrm{p}$

\begin{tabular}{|c|c|c|c|c|c|c|c|c|c|c|c|}
\hline \multirow{2}{*}{$\begin{array}{l}\text { Test } \\
\text { Samples }\end{array}$} & \multirow[b]{2}{*}{ BA } & \multirow[b]{2}{*}{$30 \mathrm{~min}$} & \multirow[b]{2}{*}{$60 \mathrm{~min}$} & \multirow[b]{2}{*}{$90 \mathrm{~min}$} & \multirow[b]{2}{*}{$120 \mathrm{~min}$} & \multirow[b]{2}{*}{$180 \mathrm{~min}$} & \multicolumn{5}{|c|}{$\%$ of inhibition } \\
\hline & & & & & & & $\begin{array}{c}30 \\
\min \end{array}$ & $\begin{array}{l}60 \\
\min \end{array}$ & $\begin{array}{c}90 \\
\min \end{array}$ & $\begin{array}{l}120 \\
\min \end{array}$ & $\begin{array}{l}180 \\
\min \end{array}$ \\
\hline Control & $5.29 \pm 0.15$ & $6.5 \pm 0.12$ & $6.3 \pm 0.15$ & $6.31 \pm 0.19$ & $6.28 \pm .0 .20$ & $6.20 \pm 0.24$ & - & - & - & - & - \\
\hline $\begin{array}{l}\text { Std. } \\
\text { (Diclofenac) }\end{array}$ & $6.37 \pm 0.09$ & $18.01 \pm 0.17$ & $26.28 \pm 0.18$ & $39.30 \pm 0.31$ & $42.37 \pm 0.39$ & $43.19 \pm 0.26$ & 30.48 & 45.70 & 57.83 & 69.30 & 90.28 \\
\hline $5 d$ & $6.14 \pm 0.10^{\text {ns }}$ & $13.53 \pm 0.17^{\#}$ & $42.35 \pm 0.28^{\#}$ & $36.20 \pm 0.13^{\#}$ & $49.18 \pm 0.26^{\#}$ & $40.03 \pm 0.25^{\#}$ & 28.59 & 42.60 & 54.72 & 65.80 & 81.60 \\
\hline $5 m$ & $6.05 \pm 0.26^{\star}$ & $11.17 \pm 0.19^{* \alpha}$ & $44.50 \pm 0.24^{\alpha \#}$ & $32.13 \pm 0.28^{\#}$ & $38.35 \pm 0.42^{\#}$ & $38.09 \pm 0.30^{\#}$ & 32.80 & 47.50 & 63.70 & 74.30 & 91.74 \\
\hline $5 n$ & $6.01 \pm 0.18^{\mathrm{ns}}$ & $14.01 \pm 0.15^{\#}$ & $45.12 \pm 0.30^{\#}$ & $34.19 \pm 0.32^{\#}$ & $49.28 \pm 0.39 \#$ & $42.04 \pm 0.27^{\alpha \#}$ & 31.50 & 43.60 & 61.80 & 68.75 & 83.49 \\
\hline 50 & $7.91 \pm 0.42^{\#}$ & $15.21 \pm 0.23^{\#}$ & $21.26 \pm 0.20^{\#}$ & $41.09 \pm 0.22^{\#}$ & $47.12 \pm 0.37^{\#}$ & $43.12 \pm 0.41^{\#}$ & 36.50 & 53.40 & 60.85 & 78.38 & 94.20 \\
\hline $5 p$ & $7.18 \pm 0.37^{\#}$ & $12.31 \pm 0.30^{\#}$ & $22.12 \pm 0.16^{\#}$ & $40.04 \pm 0.14^{\#}$ & $45.09 \pm 0.41^{\#}$ & $39.15 \pm 0.71^{\#}$ & 35.30 & 49.85 & 68.37 & 76.83 & 93.50 \\
\hline
\end{tabular}

of indomethacin and docked molecules orientations are shown in figure 1 . All the five docked molecules have the core benzimidazole ring stabilized by cation-pi interactions with side chain $\mathrm{NH}$ of Arg-120 and aromatic ring of benzimidazole in the active site, and the other residues Val-349 and Ala-527 are forming CH-pi interactions. Further, the Nitrogen atom of the amide in all molecules, except $\mathbf{5 n}$, is forming a hydrogen bond with side chain $\mathrm{NH}$ of Arg-120 and all the molecules are stabilized by hydrophobic interactions in the active site. The substituted aromatic phenyl group is also forming CH-pi interactions with side chain of both Leu-93 and Val-116 residues. The halogens (Chlorine and Bromine) on the phenyl ring are acting as electron withdrawing group and showing as anion character to make anion-pi interactions. These anion-pi interactions are as strong as hydrogen bond interactions [28]. The molecules $\mathbf{5 d}, \mathbf{5 m}, \mathbf{5 0}$, and $\mathbf{5 p}$ having halogens showing the good anti-inflammatory activity in the experiment, where the halogens are interacting with the aromatic amino acids by forming anion-pi interactions with Phe-357, Tyr-115 and Trp-100 in the active site. The molecules $\mathbf{5 n}$ and $\mathbf{5 p}$ with hydroxyl substitution on phenyl ring decreasing the activity when compared with the halogen containing aromatic rings. The hydroxyl group become more acidic and easily loose the hydrogen, and the oxygen anion and the halogen can involve in the resonance with aromatic ring. This will cause decrease in the activity of the molecule. From the docking studies, we have identified the binding energies and molecular binding orientation of the test molecules in the active site. The data is enclosed in the table 4 and the docking procedure of Autodock vina in reproducing the experimental binding affinity seems reliable, and therefore predicted as true positive.

\section{Conclusions}

In the present study, the target hydrazone derivatives (5a-r) have been synthesized and their structures are established with the aid of IR, ${ }^{1} \mathrm{H}$ NMR, ${ }^{13} \mathrm{C}$ NMR and mass spectral data. All the synthesized compounds were evaluated for in vitro antioxidant activity, and the results showed compounds $\mathbf{5 d}$, and $\mathbf{5 m - p}$ are more potent among the tested compounds. The highly active compounds from the antioxidant activity profile (5d, and $\mathbf{5 m}$-p) were further evaluated for in vivo anti-inflammatory and analgesic activity and the from the results, the compound $\mathbf{5 0}$ found to be the most effective against anti-inflammatory activity and analgesic activity. In understanding the SAR of the target compounds and based on the results, substituted halogen derivatives exhibited the potent biological activities, the hydroxy along with halogen substituted compounds exhibited moderate biological activities due to extended resonances. The docking results aid in understanding the interaction pattern in the active site and their binding affinities of all the tested compounds, further the results proved that the di-halo substitution at $o$ - and $p$ - positions is crucial for exhibiting the best activity.

\section{Experimental}

All the melting points were determined on a Cintex melting point apparatus and are uncorrected. Analytical TLC was performed on Merck precoated $60 \mathrm{~F}_{254}$ silica gel plates and visualization was done by exposing to UV Light. IR Spectra (KBr Pellet) was recorded on a PerkinElmer BX series FT-IR Spectrometer. ${ }^{1} \mathrm{H}$ NMR (500 and $400 \mathrm{MHz}$ ) and ${ }^{13} \mathrm{C}$ NMR (125 and $100 \mathrm{MHz}$ ) spectra were 

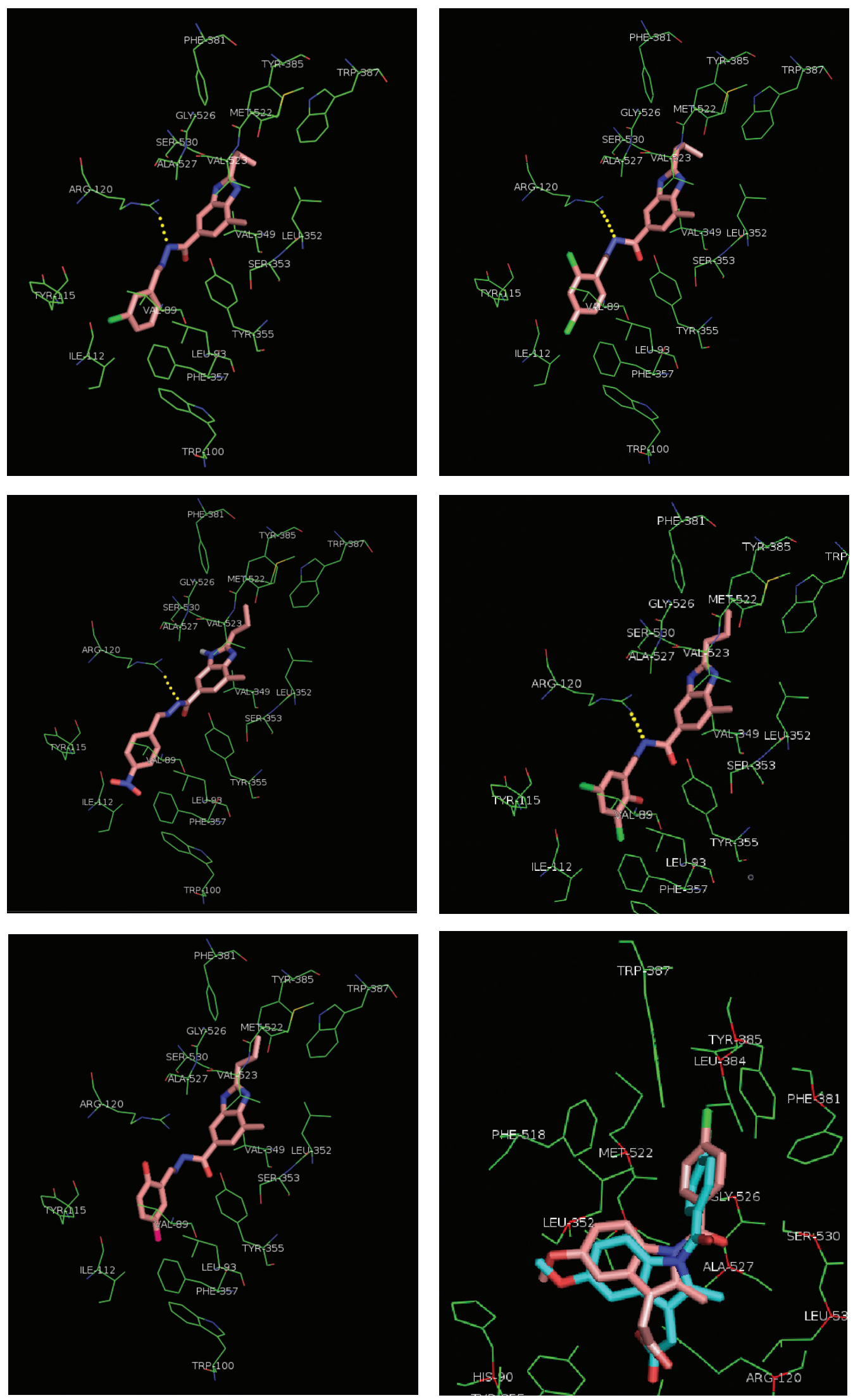

Figure 1 Molecular docking conformations of compounds 5d, 5m-p, and indomethacine (solman red, stick) into the COX-2 active site (green lines). Hydrogen bonds are shown as broken lines. 
Table 4 Binding energies of compounds $5 \mathrm{~d}$ and $5 \mathrm{~m}-\mathrm{p}$

\begin{tabular}{lc}
\hline Compound & $\begin{array}{c}\text { Binding affinity } \\
\text { (in kcal/mol) }\end{array}$ \\
\hline Std. & -8.7 \\
(Indomethacin) & -9.0 \\
5d & -8.8 \\
5m & -8.5 \\
5n & -9.3 \\
50 & -9.1 \\
\hline 5p & \\
\hline
\end{tabular}

recorded on a Bruker spectrometer in DMSO- $\mathrm{d}_{6}$ with TMS as internal standard. ESI mass spectra were recorded on an Agilent LC-MSD mass spectrometer. Elemental analyses were performed on a Carlo Erba 106 and Perkin-Elmer model 240 analysers. All the chemicals were procured from Sigma Aldrich and are used as such without further purification.

\section{Synthesis of 7-methyl-2-propyl-1H-benzo[d] imidazole- 5-carbohydrazide (3)}

A mixture of methyl 2-n-Propyl-7-methyl-benzimidazole5-carboxylate $2(0.1 \mathrm{mmol})$ in ethanol $(20 \mathrm{~mL})$ and hydrazine hydrate $(0.5 \mathrm{mmol})(98 \%)$ was heated for reflux for $10 \mathrm{~h}$, and the progress of the reaction was monitored by TLC (10\% Methanol in Dichloromethane). The reaction mixture was cooled to room temperature and the total volume of reaction mass reduced to half under reduced pressure and further cooled to $0-5^{\circ} \mathrm{C}$. The precipitated solid was filtered and crystallized from ethanol to yield carbohydrazide (3), Yield 90\%; mp 122-123우; Off White Color solid; IR: $v_{\max } 1591,1643,3124,3250,3300 \mathrm{~cm}^{-1}$; ${ }^{1} \mathrm{H}$

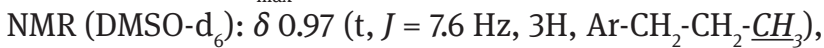
$1.75\left(\mathrm{~m}, 2 \mathrm{H}, \mathrm{Ar}-\mathrm{CH}_{2}-\mathrm{CH}_{2}-\mathrm{CH}_{3}\right), 2.60\left(\mathrm{~s}, 3 \mathrm{H}, \mathrm{Ar}-\mathrm{CH}_{3}\right), 2.80(\mathrm{t}$, $\left.J=7.6 \mathrm{~Hz}, 2 \mathrm{H}, \mathrm{Ar}-\mathrm{CH}_{2}-\mathrm{CH}_{2}-\mathrm{CH}_{3}\right), 4.49\left(\mathrm{~s}, 2 \mathrm{H}, \mathrm{NH}_{2}\right), 7.49(\mathrm{~s}$, 1H, Ar-H), 7.80 (s, 1H, Ar-H), 9.70 (s, 1H, NH), 12.25 (s, 1H, $\mathrm{NH}) ;{ }^{13} \mathrm{C}$ NMR (DMSO-d $)$ ): $\delta 15.27,19.89,21.00,39.14,113.64$, 117.67, 125.77, 133.58, 136.41, 146.07, 167.88, 172.45; MS: $\mathrm{m} / z$ $233(\mathrm{M}+\mathrm{H})^{+}$. LC-MS: $233.00(\mathrm{M}+\mathrm{H})^{+}(91.30 \%)$. Anal. Calcd for $\mathrm{C}_{12} \mathrm{H}_{16} \mathrm{~N}_{4} \mathrm{O}$ : C, 62.05; H, 6.94; N, 24.12; Found: C, 62.13; H, 6.99; N, 24.16.

\section{General procedure for synthesis of $(E)$ - $N$ '-benzylidene-7- methyl-2-propyl-1H-benzo[d] ]imidazole-5-carbohydrazide 5a-r}

A mixture of hydrazide $3(0.1 \mathrm{mmol})$ and an aromatic aldehyde (0.1 mmol) was heated under reflux in methanol (20 $\mathrm{mL}$ ) in the presence of cat. amount of glacial acetic acid for 4-6 $\mathrm{h}$. The progress of the reaction was monitored by
TLC (10\% Methanol in Dichloromethane). The reaction mixture was cooled, and the separated solid was filtered and crystallized from methanol.

\section{(E)-N'-Benzylidene-7-methyl-2-propyl-1H-benzo[d] imidazole-5-carbohydrazide (5a)}

Yield 90\%; mp 149-150우 Colorless solid; IR: $v_{\max } 3317$ $(\mathrm{NH}), 1648(\mathrm{CO}), 1552(\mathrm{C}=\mathrm{N}) \mathrm{cm}^{-1} ;{ }^{1} \mathrm{H}$ NMR (DMSO-d ${ }_{6}$ ): $\delta 0.97\left(\mathrm{t}, J=7.6 \mathrm{~Hz}, 3 \mathrm{H}, \mathrm{Ar}-\mathrm{CH}_{2}-\mathrm{CH}_{2}-\mathrm{CH}_{3}\right), 1.82(\mathrm{~m}, 2 \mathrm{H}$, Ar- $\mathrm{CH}_{2}-\mathrm{CH}_{2}-\mathrm{CH}_{3}$ ), $2.57\left(\mathrm{~s}, 3 \mathrm{H}, \mathrm{Ar}-\mathrm{CH}_{3}\right), 2.85(\mathrm{t}, J=7.6 \mathrm{~Hz}$, 2H, Ar- $\left.\mathrm{CH}_{2}-\mathrm{CH}_{2}-\mathrm{CH}_{3}\right), 7.46$ (m, 3H, Ar-H), 7.60 (s, 1H, Ar-H), 7.75 (m, 2H, Ar-H), 7.98 (s, 1H, Ar-H), 8.51 (s, 1H, - $\mathrm{CH}=\mathrm{N}-$ ) , 11.83 (s, $1 \mathrm{H}, \mathrm{NH}), 12.50$ (s, $1 \mathrm{H}, \mathrm{NH}$ ); ${ }^{13} \mathrm{C}$ NMR (DMSO-d $): \delta$ 14.17, 17.53, 21.55, 31.06, $112.60121 .95,126.76,127.45,127.70$, 129.29, 130.34, 133.02, 135.03, 147.45, 157.33, 164.38,172.53; MS: $m / z 321(\mathrm{M}+\mathrm{H})^{+}$. LC-MS: $m / z 321.12(\mathrm{M}+\mathrm{H})^{+}(95.06 \%)$. Anal. Calcd for $\mathrm{C}_{19} \mathrm{H}_{20} \mathrm{~N}_{4} \mathrm{O}: \mathrm{C}, 71.23 ; \mathrm{H}, 6.29 ; \mathrm{N}, 17.49$; Found: C, 71.30; H, 6.31; N, 17.45.

\section{(E)-N'-(4-Methoxybenzylidene)-7-methyl-2-propyl-1H- benzo[d]imidazole-5-carbohydrazide (5b)}

Yield 91\%; mp 178-179으; Colorless solid; IR: $v_{\max } 3211(\mathrm{NH})$, $1654(\mathrm{CO}), 1570(\mathrm{C}=\mathrm{N}) \mathrm{cm}^{-1} ;{ }^{1} \mathrm{H}$ NMR (DMSO-d $\left.{ }_{6}\right): \delta 0.98(\mathrm{t}, J=$ $\left.7.6 \mathrm{~Hz}, 3 \mathrm{H}, \mathrm{Ar}-\mathrm{CH}_{2}-\mathrm{CH}_{2}-\mathrm{CH}_{3}\right), 1.76\left(\mathrm{~m}, 2 \mathrm{H}, \mathrm{Ar}-\mathrm{CH}_{2}-\mathrm{CH}_{2}-\mathrm{CH}_{3}\right)$, $2.58\left(\mathrm{~s}, 3 \mathrm{H}, \mathrm{Ar}-\mathrm{CH}_{3}\right), 2.84\left(\mathrm{t}, \mathrm{J}=7.6 \mathrm{~Hz}, 2 \mathrm{H}, \mathrm{Ar}-\mathrm{CH}_{2}-\mathrm{CH}_{2}-\mathrm{CH}_{3}\right.$ ), $3.80\left(\mathrm{~s}, 3 \mathrm{H}, \mathrm{OCH}_{3}\right), 6.99$ (d, J = 8.0 Hz, 2H, Ar-H,), 7.60 (s, 1H, Ar-H), 7.70 (d, J = 8.0 Hz, 2H, Ar-H,), 7.79 (s, 1H, Ar-H), 8.49 (s, $1 \mathrm{H},-\mathrm{CH}=\mathrm{N}-), 11.60(\mathrm{~s}, 1 \mathrm{H}, \mathrm{NH}), 12.01(\mathrm{~s}, 1 \mathrm{H}, \mathrm{NH}) ;{ }^{13} \mathrm{C}$ NMR (DMSO-d $)$ ): $\delta 14.02,18.00,21.98,31.27,56.01,113.20$, 115.21, 122.0, 126.78, 127.04, 127.92, 131.20, 135.72, 147.92, 157.42, 159.90, 163.98, 171.22; MS: $m / z 351(\mathrm{M}+\mathrm{H})^{+}$. LC-MS: $\mathrm{m} / z 351.16(\mathrm{M}+\mathrm{H})^{+}(95.51 \%)$. Anal. Calcd for $\mathrm{C}_{20} \mathrm{H}_{22} \mathrm{~N}_{4} \mathrm{O}_{2}: \mathrm{C}$, 68.55; H, 6.33; N, 15.99; Found: C, 68.62; H, 6.35; N, 15.94.

\section{(E)-N'-(2-Hydroxybenzylidene)-7-methyl-2-propyl-1H- benzo[d]imidazole-5-carbohydrazide (5c)}

Yield 90\%; mp 167-168우 C Colorless solid; IR: $v_{\max } 3442$ $(\mathrm{OH}), 3233(\mathrm{NH}), 1629(\mathrm{CO}), 1559(\mathrm{C}=\mathrm{N}) \mathrm{cm}^{-1}$; ${ }^{1} \mathrm{H}$ NMR

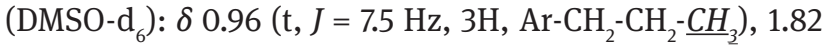
(m, 2H, Ar- $\left.\mathrm{CH}_{2}-\mathrm{CH}_{2}-\mathrm{CH}_{3}\right), 2.55$ (s, 3H, Ar-CH $), 2.82(\mathrm{t}, J=$ $\left.7.5 \mathrm{~Hz}, 2 \mathrm{H}, \mathrm{Ar}-\mathrm{CH}_{2}-\mathrm{CH}_{2}-\mathrm{CH}_{3}\right), 7.30(\mathrm{~m}, 2 \mathrm{H}, \mathrm{Ar}-\mathrm{H}), 7.55$ (s, $1 \mathrm{H}$, $\mathrm{Ar}-\mathrm{H}), 7.81$ (m, 2H, Ar-H), 7.94 (s, 1H, Ar-H), 8.48 (s, 1H, - $\mathrm{CH}=\mathrm{N}-$ ), 11.78 (s, 1H, NH), 11.98 (s, 1H, Ar-OH), 12.42 (s, $1 \mathrm{H}, \mathrm{NH}) ;{ }^{13} \mathrm{C}$ NMR (DMSO-d $)$ ) $\delta 13.68,16.83,21.04,30.57$, 113.02, 115.78, 121.12, 122.31, 119.72, 126.22, 129.05, 131.17, 133.06, 135.06, 145.743, 156.812, 159.99, 163.80, 172.00; MS: $m / z 337(\mathrm{M}+\mathrm{H})^{+}$. LC-MS: $m / z 337.28(\mathrm{M}+\mathrm{H})^{+}(97.08 \%)$. Anal. 
Calcd for $\mathrm{C}_{19} \mathrm{H}_{20} \mathrm{~N}_{4} \mathrm{O}_{2}$ : C, 67.84; H, 5.99; N, 16.66; Found: C, 67.89; H, 5.96; N, 16.69.

(E)-N'-(3-Chlorobenzylidene)-7-methyl-2-propyl-1Hbenzo[d]imidazole-5-carbohydrazide (5d)

Yield 89\%; mp 210-211우영 Off White color solid; IR: $v_{\max }$ $3317(\mathrm{NH}), 1648(\mathrm{CO}), 1552(\mathrm{C}=\mathrm{N}) \mathrm{cm}^{-1}$; ${ }^{1} \mathrm{H}$ NMR (DMSO$\left.\mathrm{d}_{6}\right): \delta 0.96\left(\mathrm{t}, J=7.6 \mathrm{~Hz}, 3 \mathrm{H}, \mathrm{Ar}-\mathrm{CH}_{2}-\mathrm{CH}_{2}-\mathrm{CH}_{3},\right), 1.81(\mathrm{~m}, 2 \mathrm{H}$, $\left.\mathrm{Ar}-\mathrm{CH}_{2}-\mathrm{CH}_{2}-\mathrm{CH}_{3}\right), 2.55\left(\mathrm{~s}, 3 \mathrm{H}, \mathrm{Ar}-\mathrm{CH}_{3}\right), 2.83(\mathrm{t}, J=7.6 \mathrm{~Hz}, 2 \mathrm{H}$, Ar- $\left.\mathrm{CH}_{2}-\mathrm{CH}_{2}-\mathrm{CH}_{3}\right), 7.54(\mathrm{~m}, 3 \mathrm{H}, \mathrm{Ar}-\mathrm{H}), 7.75(\mathrm{~d}, J=8.0 \mathrm{~Hz}, 2 \mathrm{H}$, Ar-H), 7.93 (s, 1H, Ar-H, ), 8.47(s, 1H, CH=N), 11.84 (s, 1H, $\mathrm{NH}), 12.43$ (s, $1 \mathrm{H}, \mathrm{NH}) ;{ }^{13} \mathrm{C}$ NMR (DMSO-d $)$ ): $\delta 13.67,16.80$, 21.03, 30.56, 112.80, 122.01, 126.13, 125.98, 128.55, 128.90, 131.53, 133.52, 133.72, 134.23, 136.82, 145.51, 156.80, 163.80, 171.98; MS: $m / z 355(\mathrm{M}+\mathrm{H})^{+}$. Anal. Calcd for $\mathrm{C}_{19} \mathrm{H}_{19} \mathrm{ClN}_{4} \mathrm{O}: \mathrm{C}$, 64.31; H, 5.40; N, 15.79; C, 64.38; H, 5.43; N, 15.81.

\section{(E)- $N^{\prime}$-(3-Bromobenzylidene)-7-methyl-2-propyl-1H- benzo[d]imidazole-5-carbohydrazide (5e)}

Yield 88\%; mp 173-174으; Light Brown color solid; IR: $v_{\max }$ $3308(\mathrm{NH}), 1646(\mathrm{CO}), 1548(\mathrm{C}=\mathrm{N}) \mathrm{cm}^{-1}$; ${ }^{1} \mathrm{H}$ NMR (DMSO$\left.\mathrm{d}_{6}\right): \delta 0.95\left(\mathrm{t}, J=7.6 \mathrm{~Hz}, 3 \mathrm{H}, \mathrm{Ar}-\mathrm{CH}_{2}-\mathrm{CH}_{2}-\underline{C H}_{3}\right), 1.80(\mathrm{~m}, 2 \mathrm{H}$, Ar- $-\mathrm{CH}_{2}-\mathrm{CH}_{2}-\mathrm{CH}_{3}$ ), 2.56 (s, 3H, Ar- $\left.\mathrm{CH}_{3}\right), 2.85(\mathrm{t}, J=7.6 \mathrm{~Hz}, 2 \mathrm{H}$, $\mathrm{Ar}-\mathrm{CH}_{2}-\mathrm{CH}_{2}-\mathrm{CH}_{3}$ ), 7.24 (m, 2H, Ar-H), 7.51 (m, 1H, Ar-H), 7.58 (s, 1H, Ar-H), 7.59 (m, 1H, Ar-H), 8.09 (s, 1H, Ar-H), 8.46 (s, $1 \mathrm{H}, \mathrm{CH}=\mathrm{N}), 11.68(\mathrm{~s}, 1 \mathrm{H}, \mathrm{NH}), 12.57(\mathrm{~s}, 1 \mathrm{H}, \mathrm{NH}) ;{ }^{13} \mathrm{C} \mathrm{NMR}$ (DMSO-d $\mathrm{d}_{6}$ : $\delta 13.99,17.01,21.67,31.06,113.01,122.37,124.06$, 125.98, 128.01, 128.06, 129.21, 132.06, 133.08, 135.00, 136.92, 148.02, 157.09, 163.07, 172.94; MS: $m / z 399(\mathrm{M}+\mathrm{H})^{+}$. LC-MS: $\mathrm{m} / z 399.35(\mathrm{M}+\mathrm{H})^{+}(96.68 \%)$. Anal. Calcd for $\mathrm{C}_{19} \mathrm{H}_{19} \mathrm{BrN}_{4} \mathrm{O}$ : C, 57.15; H, 4.80; N, 14.03; Found: C, 57.20; H, 4.84; N, 14.07.

\section{(E)-N'-(3-Methoxybenzylidene)-7-methyl-2-propyl-1H-} benzo[d]imidazole-5-carbohydrazide (5f)

Yield 89\%; mp 152-153우; Cream Color solid; IR: $v_{\max } 3206$ (NH), $1642(\mathrm{CO}), 1557(\mathrm{C}=\mathrm{N}) \mathrm{cm}^{-1} ;{ }^{1} \mathrm{H}$ NMR (DMSO-d 6 ): $\delta 0.94$ (t, $\left.J=7.6 \mathrm{~Hz}, 3 \mathrm{H}, \mathrm{Ar}-\mathrm{CH}_{2}-\mathrm{CH}_{2}-\mathrm{CH}_{3}\right), 1.80\left(\mathrm{~m}, 2 \mathrm{H}, \mathrm{Ar}-\mathrm{CH}_{2}-\mathrm{CH}_{2}-\right.$ $\left.\mathrm{CH}_{3}\right), 2.54\left(\mathrm{~s}, 3 \mathrm{H}, \mathrm{Ar}-\mathrm{CH}_{3}\right), 2.80\left(\mathrm{t}, J=7.6 \mathrm{~Hz}, 2 \mathrm{H}, \mathrm{Ar}-\mathrm{CH}_{2}-\mathrm{CH}_{2}^{-}\right.$ $\mathrm{CH}_{3}$ ), 3.80 (s, 3H, $\left.\mathrm{OCH}_{3}\right), 6.99(\mathrm{~d}, J=6.80 \mathrm{~Hz}, 1 \mathrm{H}, \mathrm{Ar}-\mathrm{H}), 7.27$ (m, 2H, Ar-H), 7.38 (m, 1H, Ar-H), 7.50 (s, 1H, Ar-H), 7.90 (s, $1 \mathrm{H}, \mathrm{Ar}-\mathrm{H}), 8.43(\mathrm{~s}, 1 \mathrm{H}, \mathrm{CH}=\mathrm{N}), 11.75(\mathrm{~s}, 1 \mathrm{H}, \mathrm{NH}), 12.40(\mathrm{~s}, 1 \mathrm{H}$, $\mathrm{NH}) ;{ }^{13} \mathrm{C}$ NMR (DMSO-d $): \delta 14.02,17.17,21.46,30.78,55.59$, 111.46, 113.01, 116.74, 120.66, 121.99, 125.71, 126.58, 130.42, 136.21, 136.82, 147.73, 157.54, 159.94, 164.63, 169.97; MS: $m / z 351(\mathrm{M}+\mathrm{H})^{+}$LC-MS: $m / z 351.34(\mathrm{M}+\mathrm{H})^{+}(99.07 \%)$. Anal. Calcd for $\mathrm{C}_{20} \mathrm{H}_{22} \mathrm{~N}_{4} \mathrm{O}_{2}$ : C, 68.55; H, 6.33; N, 15.99; Found: C, 68.59; H, 6.35; N, 15.97.
(E)-N'-(3,4-Dimethoxybenzylidene)-7-methyl-2-propyl$1 \mathrm{H}$-benzo[d]imidazole-5-carbohydrazide (5g)

Yield: 90\%; mp 162-163우; Colorless solid; IR: $v_{\max } 3199$ (NH), $1642(\mathrm{CO}), 1560(\mathrm{C}=\mathrm{N}) \mathrm{cm}^{-1}$; ${ }^{1} \mathrm{H}$ NMR (DMSO-d $)$ : $\delta 0.96\left(\mathrm{t}, J=7.6 \mathrm{~Hz}, 3 \mathrm{H}, \mathrm{Ar}-\mathrm{CH}_{2}-\mathrm{CH}_{2}-\mathrm{CH}_{3}\right), 1.81(\mathrm{~m}, 2 \mathrm{H}$, Ar- $\left.\mathrm{CH}_{2}-\mathrm{CH}_{2}-\mathrm{CH}_{3}\right), 2.55$ (s, 3H, Ar- $\left.\mathrm{CH}_{3}\right), 2.81(\mathrm{t}, J=7.6 \mathrm{~Hz}, 2 \mathrm{H}$, $\left.\mathrm{Ar}-\mathrm{CH}_{2}-\mathrm{CH}_{2}-\mathrm{CH}_{3}\right), 3.89\left(\mathrm{~s}, 3 \mathrm{H}, \mathrm{OCH}_{3}\right), 3.91\left(\mathrm{~s}, 3 \mathrm{H}, \mathrm{OCH}_{3}\right), 6.97$ ( m, 1H, Ar-H ), 7.26 (m, 2H, Ar-H), 7.52 (s, 1H, Ar-H), 8.02 (s, 1H, Ar-H), 8.39 (s, 1H, -CH=N-), 11.26 (s, 1H, NH,), 12.38 (s, $1 \mathrm{H}, \mathrm{NH}) ;{ }^{13} \mathrm{C}$ NMR (DMSO-d $)$ ): $\delta 13.92,17.23,21.64,31.00$, 55.58, 55.60, 113.06, 114.20, 115.98 116.20, 122.38, 124.00, 125.58, 128.78, 127.98, 137.56, 146.72, 153.20, 153.92, 163.82, 171.62; MS: $m / z 381(\mathrm{M}+\mathrm{H})^{+}$. LC-MS: $m / z 381.44[\mathrm{M}+\mathrm{H}]^{+}$ (98.68\%). Anal. Calcd for $\mathrm{C}_{21} \mathrm{H}_{24} \mathrm{~N}_{4} \mathrm{O}_{3}: \mathrm{C}, 66.30 ; \mathrm{H}, 6.36 ; \mathrm{N}$, 14.73; Found: C, 66.35; H, 6.38; N, 14.76.

\section{(E)-N'-(4-Cyanobenzylidene)-7-methyl-2-propyl-1H-} benzo[ $d]$ imidazole-5-carbohydrazide (5h)

Yield 92\%; mp 174-175우; Cream color solid; IR: $v_{\max } 3396$ (NH), 2224 (CN), 1663 (CO), 1544 (C=N) cm ${ }^{-1}$; ${ }^{1} \mathrm{H}$ NMR (DMSO$\left.\mathrm{d}_{6}\right): \delta 0.97\left(\mathrm{t}, J=7.5 \mathrm{~Hz}, 3 \mathrm{H}, \mathrm{Ar}-\mathrm{CH}_{2}-\mathrm{CH}_{2}-\underline{C H}_{3}\right), 1.82(\mathrm{~m}, 2 \mathrm{H}$, $\mathrm{Ar}-\mathrm{CH}_{2}-\mathrm{CH}_{2}-\mathrm{CH}_{3}$ ), 2.55 (s, 3H, Ar-CH$), 2.84$ (t, $J=7.5 \mathrm{~Hz}, 2 \mathrm{H}$, $\mathrm{Ar}-\mathrm{CH}_{2}-\mathrm{CH}_{2}-\mathrm{CH}_{3}$ ), 7.57 (s, 1H, Ar-H), 7.91 (s, 4H, Ar-H), 7.95 (s, 1H, Ar-H), 8.53 (s, 1H, CH=N), $12.02(\mathrm{~s}, 1 \mathrm{H}, \mathrm{NH}), 12.40$ (s, $1 \mathrm{H}, \mathrm{NH}) ;{ }^{13} \mathrm{C}$ NMR (DMSO-d $\left.\mathrm{d}_{6}\right): \delta 13.70,16.80,21.02,30.55$, 111.63, 114.21, 122.21, 118.67, 125.91, 127.47, 132.72, 135.02, 139.06, , 144.80, 148.90 156.90, 163.95, 171.20; MS: $\mathrm{m} / z 346$ $(\mathrm{M}+\mathrm{H})^{+}$. Anal. Calcd for $\mathrm{C}_{20} \mathrm{H}_{19} \mathrm{~N}_{5} \mathrm{O}: \mathrm{C}, 69.55 ; \mathrm{H}, 5.54 ; \mathrm{N}$, 20.28; Found: C, 69.62; H, 5.59; N, 20.33.

\section{(E)-N'-((Furan-2-yl)methylene)-7-methyl-2-propyl-1H- benzo[d]imidazole-5-carbohydrazide (5i)}

Yield 85\%; mp 170-171으; Off White color solid; IR: $v_{\max } 3234$ (NH), 1637(CO), 1556 (C=N) cm ${ }^{-1}$; ${ }^{1} \mathrm{H}$ NMR (DMSO-d $): \delta 0.95$ $\left(\mathrm{t}, J=7.6 \mathrm{~Hz}, 3 \mathrm{H}, \mathrm{Ar}-\mathrm{CH}_{2}-\mathrm{CH}_{2}-\mathrm{CH}_{3}\right), 1.78\left(\mathrm{~m}, 2 \mathrm{H}, \mathrm{Ar}^{-} \mathrm{CH}_{2}-\mathrm{CH}_{2}-\right.$ $\left.\mathrm{CH}_{3}\right), 2.55\left(\mathrm{~s}, 3 \mathrm{H}, \mathrm{Ar}-\mathrm{CH}_{3}\right), 2.82\left(\mathrm{t}, J=7.6 \mathrm{~Hz}, 2 \mathrm{H}, \mathrm{Ar}-\mathrm{CH}_{2}-\mathrm{CH}_{2}-\right.$ $\mathrm{CH}_{3}$ ), 7.59-7.70 (m, 4H, Ar-H), $8.02(\mathrm{~s}, 1 \mathrm{H}, \mathrm{Ar}-\mathrm{H}), 9.18(\mathrm{~s}, 1 \mathrm{H}$, $\mathrm{CH}=\mathrm{N}$ ) , $11.82(\mathrm{~s}, 1 \mathrm{H}, \mathrm{NH}), 12.41(\mathrm{~s}, 1 \mathrm{H}, \mathrm{NH}) ;{ }^{13} \mathrm{C}$ NMR (DMSO$\left.\mathrm{d}_{6}\right): \delta 14.08,17.52,21.48,30.88,113.10,121.92,124.57,126.08$, 126.75, 133.97, 136.00, 143.5, 147.27, 155.01, 157.27, 164.05, 170.20; MS: $m / z 311(\mathrm{M}+\mathrm{H})^{+}$. Anal. Calcd for $\mathrm{C}_{17} \mathrm{H}_{18} \mathrm{~N}_{4} \mathrm{O}_{2}: \mathrm{C}$, 65.79; H, 5.85; N, 18.05; Found: C, C, 65.84; H, 5.88; N, 18.06.

(E)-7-Methyl-2-propyl-N'-((thiophen-2-yl)methylene)-1Hbenzo[d]imidazole-5-carbohydrazide (5j)

Yield 90\%; mp 175-176으; Light Brown color solid; IR: $v_{\max }$ $3186(\mathrm{NH}), 1643(\mathrm{CO}), 1558(\mathrm{C}=\mathrm{N}) \mathrm{cm}^{-1} ;{ }^{1} \mathrm{H}$ NMR $(\text { DMSO-d })_{6}$ ): 
$\delta 0.99\left(\mathrm{t}, J=7.6 \mathrm{~Hz}, 3 \mathrm{H}, \mathrm{Ar}-\mathrm{CH}_{2}-\mathrm{CH}_{2}-\mathrm{CH}_{3}\right), 1.85(\mathrm{~m}, 2 \mathrm{H}$, $\mathrm{Ar}-\mathrm{CH}_{2}-\mathrm{CH}_{2}-\mathrm{CH}_{3}$ ), 2.58 (s, 3H, Ar- $-\mathrm{CH}_{3}$ ), 2.80 (t, $J=7.6 \mathrm{~Hz}$, $\left.2 \mathrm{H}, \mathrm{Ar}-\mathrm{CH}_{2}-\mathrm{CH}_{2}-\mathrm{CH}_{3}\right), 7.15(\mathrm{~m}, 1 \mathrm{H}, \mathrm{Ar}-\mathrm{H}), 7.47(\mathrm{~m}, 1 \mathrm{H}, \mathrm{Ar}-\mathrm{H})$, 7.50 (s, 1H, Ar-H), 7.62 (m, 1H, Ar-H), 7.99 (s, 1H, Ar-H), 8.74 $(\mathrm{s}, 1 \mathrm{H}, \mathrm{CH}=\mathrm{N}), 11.75(\mathrm{~s}, 1 \mathrm{H}, \mathrm{NH}), 12.00(\mathrm{~s}, 1 \mathrm{H}, \mathrm{NH}) ;{ }^{13} \mathrm{C}$ NMR

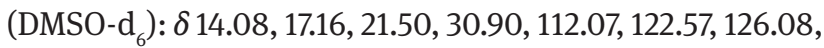
127.24, 130.10, 130.67, 133.97, 136.02, 145.20, 147.17, 157.07, 163.02, 171.62; MS: $m / z 327(\mathrm{M}+\mathrm{H})^{+}$. Calcd for $\mathrm{C}_{17} \mathrm{H}_{18} \mathrm{~N}_{4} \mathrm{OS}$ : C, 62.55; H, 5.56; N, 17.16; Found: C, 62.60; H, 5.59; N, 17.18.

(E)-7-Methyl- $N$ '-((naphthalen-2-yl)methylene)-2-propyl$1 \mathrm{H}$-benzo[d]imidazole-5-carbohydrazide (5k)

Yield 90\%; mp 191-192ㅇ; Colorless solid; IR: $v_{\max } 3317(\mathrm{NH})$, $1648(\mathrm{CO}), 1542(\mathrm{C}=\mathrm{N}) \mathrm{cm}^{-1} ;{ }^{1} \mathrm{H}$ NMR (DMSO-d $): \delta 0.98(\mathrm{t}, J=$ $7.6 \mathrm{~Hz}, 3 \mathrm{H}, \mathrm{Ar}-\mathrm{CH}_{2}-\mathrm{CH}_{2}-\mathrm{CH}_{3}$ ), 1.79 (m, $2 \mathrm{H}, \mathrm{Ar}-\mathrm{CH}_{2}-\mathrm{CH}_{2}-\mathrm{CH}_{3}$ ), $2.57\left(\mathrm{~s}, 3 \mathrm{H}, \mathrm{Ar}-\mathrm{CH}_{3}\right), 2.80$ (t, $\left.J=7.6 \mathrm{~Hz}, 2 \mathrm{H}, \mathrm{Ar}-\mathrm{CH}_{2}-\mathrm{CH}_{2}-\mathrm{CH}_{3}\right)$, 7.40-7.88 (m, 9H, Ar-H), 8.39 (s, 1H, CH=N), 11.77 (s, 1H, $\mathrm{NH}), 12.40$ (s, 1H, NH); ${ }^{13} \mathrm{C}$ NMR (DMSO-d $)$ ) $\delta$ 13.97, 17.57, 21.84, 31.20, 113.82, 122.08, 125.92, 126.04, 126.61, 127.22, 128.01, 128.82, 130.41, 131.22, 134.02, 135.82, 136.00, 147.82, 156.92, 163.29, 172.08; MS: $m / z 371(\mathrm{M}+\mathrm{H})^{+}$. LC-MS: $m / z$ $371.37(\mathrm{M}+\mathrm{H})^{+}(99.22 \%)$. Anal. Calcd for $\mathrm{C}_{23} \mathrm{H}_{22} \mathrm{~N}_{4} \mathrm{O}: \mathrm{C}, 74.57$; H, 5.99; N, 15.12; Found: C, 74.59; H, 5.98; N, 15.14 .

\section{7-Methyl-N'-[(E)-(2-methylphenyl)methylidene]-2-pro- pyl-1H-benzimidazole-5-carbohydrazide (5l)}

Yield 91\%; mp 165-166우; Colorless solid; IR: $v_{\max } 3199(\mathrm{NH})$, 1609 (CO), 1551(C=N) cm ${ }^{-1}$; ${ }^{1} \mathrm{H}$ NMR (DMSO-d $\left.\mathrm{d}_{6}\right): \delta 0.95$ (t, $J=$ $\left.7.6 \mathrm{~Hz}, 3 \mathrm{H}, \mathrm{Ar}-\mathrm{CH}_{2}-\mathrm{CH}_{2}-\mathrm{CH}_{3}\right), 1.80\left(\mathrm{~m}, 2 \mathrm{H}, \mathrm{Ar}-\mathrm{CH}_{2}-\mathrm{CH}_{2}-\mathrm{CH}_{3}\right.$ ), 2.44 (s, 3H, $\mathrm{Ar}^{-\mathrm{CH}_{3}}$ ), 2.56 (s, 3H, $\left.\mathrm{Ar}-\mathrm{CH}_{3}\right), 2.80$ (t, $J=7.6 \mathrm{~Hz}$, $\left.2 \mathrm{H}, \mathrm{Ar}-\mathrm{CH}_{2}-\mathrm{CH}_{2}-\mathrm{CH}_{3}\right), 7.25$ (m, 3H, Ar-H), 7.56 (s, $\left.1 \mathrm{H}, \mathrm{Ar}-\mathrm{H}\right)$, 7.84 (d, $J=7.2 \mathrm{~Hz}, 1 \mathrm{H}, \operatorname{Ar}-\mathrm{H}), 7.99$ (s, $1 \mathrm{H}, \operatorname{Ar}-\mathrm{H}), 8.76$ (s, $1 \mathrm{H}$, $\mathrm{CH}=\mathrm{N}$ ), $11.75(\mathrm{~s}, 1 \mathrm{H}, \mathrm{NH}), 12.40(\mathrm{~s}, 1 \mathrm{H}, \mathrm{NH}) ;{ }^{13} \mathrm{C}$ NMR (DMSO$\left.\mathrm{d}_{6}\right): \delta 14.04,17.19,19.37,21.48,30.81,113.08,121.98,127.02$, 126.21, 126.64, 130.20, 131.28, 132.73, 135.62, 137.31, 141.92, 146.35, 157.47, 164.35, 170.92; MS: $m / z 335(\mathrm{M}+\mathrm{H})^{+}$. LC-MS: $m / z 335.33(\mathrm{M}+\mathrm{H})^{+}(99.79 \%)$. Anal. Calcd for $\mathrm{C}_{20} \mathrm{H}_{22} \mathrm{~N}_{4} \mathrm{O}: \mathrm{C}$, 71.83; H, 6.63; N, 16.75 Found: C, 71.86; H, 6.64; N, 16.77.

\section{(E)-N'-(4-Nitrobenzylidene)-7-methyl-2-propyl-1H- benzo[ $d]$ imidazole-5-carbohydrazide $(5 \mathrm{~m})$}

Yield 92\%; mp 210-211우; Yellow color solid; IR: $v_{\max } 3298$ (NH), 1655 (CO), $1557\left(\mathrm{C}=\mathrm{N}\right.$ ) cm ${ }^{-1}$; ${ }^{1} \mathrm{H}$ NMR (DMSO-d ${ }_{6}$ ): $\delta 0.96$ $\left(\mathrm{t}, J=7.6 \mathrm{~Hz}, 3 \mathrm{H}, \mathrm{Ar}-\mathrm{CH}_{2}-\mathrm{CH}_{2}-\mathrm{CH}_{3}\right), 1.80\left(\mathrm{~m}, 2 \mathrm{H}, \mathrm{Ar}-\mathrm{CH}_{2}-\mathrm{CH}_{2}-\right.$ $\left.\mathrm{CH}_{3}\right), 2.51\left(\mathrm{~s}, 3 \mathrm{H}, \mathrm{Ar}-\mathrm{CH}_{3}\right), 2.81\left(\mathrm{t}, J=7.6 \mathrm{~Hz}, 2 \mathrm{H}, \mathrm{Ar}-\mathrm{CH}_{2}-\mathrm{CH}_{2}-\right.$ $\left.\mathrm{CH}_{3}\right), 7.35$ (d, $\left.J=8.4 \mathrm{~Hz}, 2 \mathrm{H}, \mathrm{Ar}-\mathrm{H}\right), 7.52$ (s, 1H, Ar-H), 8.01 (s, $1 \mathrm{H}, \mathrm{Ar}-\mathrm{H}), 8.22(\mathrm{~d}, J=8.4 \mathrm{~Hz}, 2 \mathrm{H}, \mathrm{Ar}-\mathrm{H}), 8.34(\mathrm{~s}, 1 \mathrm{H}$, $\mathrm{CH}=\mathrm{N}), 11.92(\mathrm{~s}, 1 \mathrm{H}, \mathrm{NH}), 12.25(\mathrm{~s}, 1 \mathrm{H}, \mathrm{NH}) ;{ }^{13} \mathrm{C} \mathrm{NMR}$
(DMSO-d $)$ : $\delta 14.10,16.99,21.12,30.85,112.81,114.21,123.22$, 125.23, 126.72, 131.38, 138.52, 139.27, 146.82, 148.93, 157.23, 163.72, 170.98; MS: $m / z 366.15(\mathrm{M}+\mathrm{H})^{+}$. Anal. Calcd for $\mathrm{C}_{19} \mathrm{H}_{19} \mathrm{~N}_{5} \mathrm{O}_{3}: \mathrm{C}, 62.46 ; \mathrm{H}, 5.24 ; \mathrm{N}, 19.17$; Found: C, 62.39; H, 5.28; N, 19.19 .

\section{(E)-N'-(5-B romo-2-hydroxybenzylidene)-7-methyl-2-pro-} pyl-1H-benzo[d]imidazole-5-carbohydrazide (5n)

Yield 90\%; mp 192-193으; Off White color solid; IR: $v_{\max }$ 3409 (OH), $3222(\mathrm{NH}), 1665(\mathrm{CO}), 1548(\mathrm{C}=\mathrm{N}) \mathrm{cm}^{-1}$; ${ }^{1} \mathrm{H}$ NMR

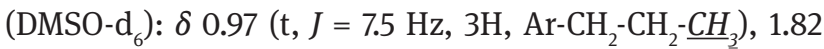
(m, $\left.2 \mathrm{H}, \mathrm{Ar}-\mathrm{CH}_{2}-\mathrm{CH}_{2}-\mathrm{CH}_{3}\right), 2.56\left(\mathrm{~s}, 3 \mathrm{H}, \mathrm{Ar}-\mathrm{CH}_{3}\right), 2.84(\mathrm{t}, J=$ $\left.7.5 \mathrm{~Hz}, 2 \mathrm{H}, \mathrm{Ar}-\mathrm{CH}_{2}-\mathrm{CH}_{2}-\mathrm{CH}_{3}\right), 6.91(\mathrm{~d}, J=8.5 \mathrm{~Hz}, 1 \mathrm{H}, \mathrm{Ar}-\mathrm{H})$, $7.43(\mathrm{dd}, J=8.5 \mathrm{~Hz}, J=2.0 \mathrm{~Hz}, 1 \mathrm{H}, \mathrm{Ar}-\mathrm{H}), 7.58(\mathrm{~s}, 1 \mathrm{H}, \mathrm{Ar}-\mathrm{H})$, $8.03(\mathrm{~s}, 1 \mathrm{H}, \mathrm{Ar}-\mathrm{H}), 7.77$ (s, 1H, Ar-H) $8.62(\mathrm{~s}, 1 \mathrm{H}, \mathrm{CH}=\mathrm{N}), 11.46$ (s, 1H, Ar-OH), 12.14 (s, 1H, NH), 12.47 (s, 1H, NH); ${ }^{13} \mathrm{C}$ NMR (DMSO-d $\mathrm{d}_{6}$ : $\delta 13.67,16.66,21.03,30.50,115.62,118.65,120.81$, 120.56, 121.90, 125.43, 126.92, 130.63, 133.30, 136.21, 145.10, 156.42, 156.81, 163.61, 173.40; MS: $m / z 415(\mathrm{M}+\mathrm{H})^{+}$. LC-MS: $\mathrm{m} / z 415.33(\mathrm{M}+\mathrm{H})^{+}(99.84 \%)$. Anal. Calcd for $\mathrm{C}_{19} \mathrm{H}_{19} \mathrm{BrN}_{4} \mathrm{O}_{2}$ : C, 54.95; H, 4.61; N, 13.49; Found: C, 54.99; H, 4.62; N, 13.51.

(E)-N'-(2,4-Dichlorobenzylidene)-7-methyl-2-propyl-1Hbenzo[d]imidazole-5-carbohydrazide (50)

Yield: 92\%; mp 201-202으; Light Brown color solid; IR: $v_{\max } 3317(\mathrm{NH}), 1648(\mathrm{CO}), 1542(\mathrm{C}=\mathrm{N}) \mathrm{cm}^{-1}$; ${ }^{1} \mathrm{H}$ NMR (DMSO$\left.\mathrm{d}_{6}\right): \delta 0.94\left(\mathrm{t}, 3 \mathrm{H}, J=7.6 \mathrm{~Hz}, \mathrm{Ar}_{-} \mathrm{CH}_{2}-\mathrm{CH}_{2}-\mathrm{CH}_{3}\right), 1.80(\mathrm{~m}, 2 \mathrm{H}$, $\mathrm{Ar}-\mathrm{CH}_{2}-\mathrm{CH}_{2}-\mathrm{CH}_{3}$ ), $2.53\left(\mathrm{~s}, 3 \mathrm{H}, \mathrm{Ar}-\mathrm{CH}_{3}\right), 2.81(\mathrm{t}, J=7.6 \mathrm{~Hz}, 2 \mathrm{H}$, $\left.\mathrm{Ar}-\mathrm{CH}_{2}-\mathrm{CH}_{2}-\mathrm{CH}_{3}\right), 7.50(\mathrm{~d}, J=8.8 \mathrm{MHz}, 1 \mathrm{H}, \mathrm{Ar}-\mathrm{H}), 7.55(\mathrm{~s}, 1 \mathrm{H}$, Ar-H), 7.68 (s, 1H, Ar-H), 7.85 (s, 1H, Ar-H), 8.01 (d, J = 7.2 $\mathrm{Hz}$ 1H, Ar-H), 8.81 (s, 1H, CH=N), 12.04 (s, 1H, NH), 12.41 (s, $1 \mathrm{H}, \mathrm{NH}) ;{ }^{13} \mathrm{C}$ NMR (DMSO-d $): \delta 14.15,17.58,21.50,31.02$, 112.82, 122.05, 126.61, 127.98, 128.44, 129.79, 131.42, 134.14, 135.26, 138.21,147.02, 157.82,158.02 164.02, 171.03; MS: $m / z$ $389(\mathrm{M}+\mathrm{H})^{+}$. LC-MS: $m / z 389.32(\mathrm{M}+\mathrm{H})^{+}(98.84 \%)$. Anal. Calcd for $\mathrm{C}_{19} \mathrm{H}_{18} \mathrm{Cl}_{2} \mathrm{~N}_{4} \mathrm{O}$ : C, 58.62; H, 4.66; N, 14.39; Found: C, 58.65; H, 4.67; N, 14.42 .

(E)-N'-(3,5-Dichloro-2-hydroxybenzylidene)-7-methyl-2propyl-1H-benzo[d]imidazole-5-carbohydrazide (5p)

Yield 90\%; mp 181-182으; Colorless solid; IR: $v_{\text {max }}$ 3202(OH), $3160(\mathrm{NH}), 1656(\mathrm{CO}), 1552(\mathrm{C}=\mathrm{N}) \mathrm{cm}^{-1}$; ${ }^{1} \mathrm{H}$ NMR (DMSO$\left.\mathrm{d}_{6}\right): \delta 0.92\left(\mathrm{t}, J=7.5 \mathrm{~Hz}, 3 \mathrm{H}, \mathrm{Ar}-\mathrm{CH}_{2}-\mathrm{CH}_{2}-\underline{\mathrm{CH}}_{3}\right), 1.80(\mathrm{~m}, 2 \mathrm{H}$, Ar- $-\mathrm{CH}_{2}-\mathrm{CH}_{2}-\mathrm{CH}_{3}$ ), 2.53 (s, 3H, Ar- $\mathrm{CH}_{3}$ ), $2.81(\mathrm{t}, J=7.5 \mathrm{~Hz}$, $\left.2 \mathrm{H}, \mathrm{Ar}-\mathrm{CH}_{2}-\mathrm{CH}_{2}-\mathrm{CH}_{3}\right), 7.57(\mathrm{~m}, 2 \mathrm{H}, \mathrm{Ar}-\mathrm{H}), 7.62(\mathrm{~d}, J=2.8 \mathrm{~Hz}$, $1 \mathrm{H}, \mathrm{Ar}-\mathrm{H}), 8.00$ (s, 1H, Ar-H), 8.58 (s, 1H, - $\mathrm{CH}=\mathrm{N}-$ ) , 12.42 (s, 2H, NH), 12.42 (s, 2H, NH), 12.68 (s, 1H, Ar-OH); ${ }^{13} \mathrm{C} \mathrm{NMR}$ $\left(\right.$ DMSO-d $\left._{6}\right): \delta 14.13,17.26,21.49,31.03,113.62,121.26,121.82$, 
123.25, 125.40, 125.99, 128.76, 128.80, 130.42, 136.99, 146.58, 152.70, 157.54, 164.11, 171.82; MS: $m / z 405(\mathrm{M}+\mathrm{H})^{+}$. LC-MS: $m / z 405.33(\mathrm{M}+\mathrm{H})^{+}(99.83 \%)$. Anal. Calcd for $\mathrm{C}_{19} \mathrm{H}_{18} \mathrm{Cl}_{2} \mathrm{~N}_{4} \mathrm{O}_{2}$ : C, 56.31; H, 4.48; N, 13.82; Found: C, 56.32; H, 4.49; N, 13.80.

\section{(E)-N'-(4-Hydroxybenzylidene)-7-methyl-2-propyl-1H-} benzo[d]imidazole-5-carbohydrazide ( $5 q)$

Yield 90\%; mp 206-207우; Colorless solid; IR: $v_{\max } 3448$ (OH), $3224(\mathrm{NH}), 1602(\mathrm{CO}), 1510(\mathrm{C}=\mathrm{N}) \mathrm{cm}^{-1}$; ${ }^{1} \mathrm{H}$ NMR (DMSO$\left.\mathrm{d}_{6}\right): \delta 0.93\left(\mathrm{t}, J=6.4 \mathrm{~Hz}, 3 \mathrm{H}, \mathrm{Ar}-\mathrm{CH}_{2}-\mathrm{CH}_{2}-\mathrm{CH}_{3}\right), 1.79(\mathrm{~m}, 2 \mathrm{H}$, $\mathrm{Ar}-\mathrm{CH}_{2}-\mathrm{CH}_{2}-\mathrm{CH}_{3}$ ), 2.52 (s, 3H, Ar- $\left.\mathrm{CH}_{3}\right), 2.80(\mathrm{t}, J=6.4 \mathrm{~Hz}$, $2 \mathrm{H}, \mathrm{Ar}-\mathrm{CH}_{2}-\mathrm{CH}_{2}-\mathrm{CH}_{3}$ ), 6.82 (d, $\left.J=6.8 \mathrm{~Hz} 2 \mathrm{H}, \mathrm{Ar}-\mathrm{H}\right), 7.52$ (bs, 3H, Ar-H), 7.89 (s, 1H, Ar-H), 8.35 (s, 1H, CH=N), 9.90 (s, 1H, Ar-OH), 11.56 (s, 1H, NH), 12.41 (s, $1 \mathrm{H}, \mathrm{NH}) ;{ }^{13} \mathrm{C}$ NMR (DMSO$\left.\mathrm{d}_{6}\right): \delta 14.15,17.29,21.51,31.02,116.14,112.88,121.82,125.99$, 126.99, 129.14, 130.42, 138.02, 146.58, 152.71, 157.54, 164.11, 172.09; MS: $m / z 337(\mathrm{M}+\mathrm{H})^{+}$. Anal. Calcd for $\mathrm{C}_{19} \mathrm{H}_{20} \mathrm{~N}_{4} \mathrm{O}_{2}: \mathrm{C}$, 67.84; H, 5.99; N, 16.66; Found: C, 67.90; H, 6.01; N, 16.70.

\section{(E)-N'-(4-Hydroxy-3-methoxybenzylidene)-7-methyl- 2-propyl-1H-benzo[d] imidazole-5-carbohydrazide (5r)}

Yield 91\%; mp 198-199우; Cream color solid; IR: $v_{\max } 3317$ (OH), $3250(\mathrm{NH}), 1650(\mathrm{CO}), 1552(\mathrm{C}=\mathrm{N}) \mathrm{cm}^{-1}$; ${ }^{1} \mathrm{H}$ NMR (DMSO$\left.\mathrm{d}_{6}\right): \delta 0.93\left(\mathrm{t}, J=7.6 \mathrm{~Hz}, 3 \mathrm{H}, \mathrm{Ar}-\mathrm{CH}_{2}-\mathrm{CH}_{2}-\mathrm{CH}_{3}\right), 1.82(\mathrm{~m}, 2 \mathrm{H}$, Ar- $\mathrm{CH}_{2}-\mathrm{CH}_{2}-\mathrm{CH}_{3}$ ), 2.52 (s, 3H, Ar- $\left.\mathrm{CH}_{3}\right), 2.84$ (t, $J=7.6 \mathrm{~Hz}, 2 \mathrm{H}$, Ar- $\left.\underline{\mathrm{CH}}_{2}-\mathrm{CH}_{2}-\mathrm{CH}_{3}\right), 3.80\left(\mathrm{~s}, 3 \mathrm{H}, \mathrm{OCH}_{3}\right), 6.76(\mathrm{~d}, J=8.5 \mathrm{~Hz}, 1 \mathrm{H}$, Ar-H), 7.15(d, J=8.5 Hz, 1H, Ar-H), 7.40 (s, 1H, Ar-H), 7.49 (s, 1H, Ar-H),7.98 (s, 1H, Ar-H), 8.30 (s, 1H, CH=N), 9.61 (s, 1H, Ar-OH), 11.59 (s, 1H, NH), 12.50 (s, 1H, Ar-OH); ${ }^{13} \mathrm{C}$ NMR (DMSO-d $)$ : $\delta$ 14.13, 17.26, 21.49, 31.03, 56.00, 112.81, 113.82, 119.02, 121.82, $123.25,125.39,128.76,129.42,136.79,146.58,147.52,152.70$, 157.54, 164.11, 171.97; MS: $m / z 367(\mathrm{M}+\mathrm{H})^{+}$. LC-MS: $m / z 367.34$ $(\mathrm{M}+\mathrm{H})^{+}(94.11 \%)$. Anal. Calcd for $\mathrm{C}_{20} \mathrm{H}_{22} \mathrm{~N}_{4} \mathrm{O}_{3}:$ C, 65.56; H, 6.05; N, 15.29; Found: C, 65.62; H, 6.08; N, 15.33.

\section{Antioxidant activity}

The in vitro antioxidant activity has been evaluated by 1,1-Diphenyl-2-picrylhydrazyl (DPPH) radical scavenging test following the literature protocol [29]. The free radical scavenging activities of 5a-r were measured by DPPH with slightly modified spectrophotometric method. A solution of DPPH in methanol $\left(6 \times 10^{-5} \mathrm{M}\right)$ was prepared freshly and $3 \mathrm{~mL}$ aliquot of this solution was mixed with $1 \mathrm{~mL}$ of the samples at varying concentrations. The solutions in the test tubes (5a-r) were shaken well and incubated in the dark for $15 \mathrm{~min}$ at room temperature. The decrease in absorbance was measured at $517 \mathrm{~nm}$. Ascorbic acid was used as the reference material. All the tests were performed in triplicate and the graph was plotted with the mean values. The percentage of inhibition was calculated by comparing the absorbance values of the control and test samples. The percentage inhibition was calculated by using the formula, $\%$ Inhibition $=\left[\left(A_{\text {control }}-A_{\text {sample }}\right) / A_{\text {control }}\right] \times 100$.

\section{Anti-inflammatory Activity}

The in vivo anti-inflammatory activity was determined by carrageenan-induced paw edema method [30]. Wistar rats of either sex weighing 150-200 g were divided into 6 groups $(n=6)$. They were fasted for $18 \mathrm{~h}$ before the experiment with water ad libitum. The groups of rats administered with $0.5 \%$ DMSO (negative control), indomethacin at a dose of $10 \mathrm{mg} / \mathrm{kg}$ (positive control), and test samples at $10 \mathrm{mg} / \mathrm{kg}$. All the compounds were administered in an oral route and after $30 \mathrm{~min}, 0.1 \mathrm{~mL}$ of $1 \%$ carrageenan suspension in normal saline was injected into the subplantar region of the left hind paw of each rat to induce edema. The edema volumes of the injected paw were measured with the help of plethysmograph at an interval of $0,1,2,4$, and $6 \mathrm{~h}$. The difference between the paw volumes of treated animals were compared with that of the control group and the mean edema volume was calculated. Percentage inhibition was calculated as per the formula, \%Inhibition $=\left[V_{o}\right.$ $\left.-V_{t} / V_{o}\right] \times 100$, where $V_{o}=$ volume of the paw control at time $t, V_{t}=$ volume of the paw of drug treated at time $t$.

\section{Analgesic activity}

The in vivo analgesic activity test was conducted using the hot-plate method [31, 32]. Albino mice weighing 20-30 gm were divided into eleven groups of six in each group. The temperature on hot plate is controlled at $55 \pm 10^{\circ} \mathrm{C}$. The animals were placed on the plate on the heated surface and the time (sec) to discomfort reaction (licking paws or jumping) was recorded as response latency period at time interval of 30, 60, 90, 120 and 180 min following oral administration of the standard and the test compounds. A latency period of $45 \mathrm{sec}$ was identified as complete analgesia and the measurement was terminated if it exceeded the latency period in order to avoid injury.

\section{Molecular docking studies:}

Molecular modelling studies of five selected compounds were carried out using Autodock vina software [33] and docking of all compounds was carried out on cyclooxygenase-2 complexed with indomethacin (PDB_ID 4COX, chain A). This protein PDB was retrieved from protein data 
bank. During the docking studies, we have used $1 \AA$ grid box parameters as centre: $\mathrm{x}=22.741, \mathrm{y}=23.808, \mathrm{z}=15.008$ and grid box size: $x=20, y=22, z=22$ and while docking 20 conformations were generated for each ligand by using default genetic algorithm. In the present study, input preparation carried out using MGL tools-1.5.6 and final docking performed in Autodock vina.

\section{References}

[1] Takaya, M.; Sato, M.; Terashima, K.; Tanizawa, H.; Maki, $Y$. A new nonsteroidal analgesic-anti-inflammatory agent. Synthesis and activity of 4-ethoxy-2-methyl-5-morpholino-3(2H)-pyridazinone and related compounds. J. Med. Chem. 1979, 22, 53-58.

[2] Heinisch, G.; Frank, H.; Ellis, G. P.; West, G. B. edition Elsevier Science B.V. Prog. Med. Chem. 1990, 27, 1.

[3] Gaba, M.; Singh, D.; Singh, S.; Sharma, V.; Gaba, P. Synthesis and pharmacological evaluation of novel 5 -substituted1-(phenylsulfonyl)-2-methylbenzimidazole derivatives as anti-inflammatory and analgesic agents. Eur. J. Med. Chem. 2010, 45, 2245-2249.

[4] Thimmegowda, N. R.; Nanjunda Swamy, S.; Ananda Kumar, C. S.; Sunil Kumar, Y. C.; Chandrappa, S.; Yip, George. W.; Rangappa, K.S. Synthesis, characterization and evaluation of benzimidazole derivative and its precursors as inhibitors of MDA-MB-231 human breast cancer cell proliferation. Bioorg. Med. Chem. Lett. 2008, 18, 432-435.

[5] Beaulieu, P. L.; Bös, M.; Bousquet, Y.; Fazal, G.; Gauthier, J.; Gillard, J.; Goulet, S.; LaPlante, S.; Poupart, M. A.; Lefebvre, S.; McKercher, G.; Pellerin, C.; Austel, V.; Kukolj, G. Non-nucleoside inhibitors of the hepatitis C virus NS5B polymerase: discovery and preliminary SAR of benzimidazole derivatives. Bioorg. Med. Chem. Lett. 2004, 14,119-124.

[6] Beaulieu, P. L.; Bös, M.; Bousquet, Y.; Deroy, P.; Fazal, G.; Gauthier, J.; Gillard, J.; Goulet, S.; McKercher, G.; Poupart, M. A.; Valois, S.; Kukolj, G. Non-nucleoside inhibitors of the hepatitis $C$ virus NS5B polymerase: discovery of benzimidazole 5-carboxylic amide derivatives with low-nanomolar potency. Bioorg. Med. Chem. Lett. 2004, 14, 967-971.

[7] Beaulieu, P. L.; Bousquet, Y.; Gauthier, J.; Gillard, J.; Marquis, M.; McKercher, G.; Pellerin, C.; Valois, S.; Kukolj, G. Non-Nucleoside Benzimidazole-Based Allosteric Inhibitors of the Hepatitis C Virus NS5B Polymerase: Inhibition of Subgenomic Hepatitis C Virus RNA Replicons in Huh-7 Cells," J. Med. Chem. 2004, 47, 6884-6892.

[8] Xue, C. B.; Rafalski, M.; Roderick, J.; Eyermann, C. J.; Mousa, S.; Olson, R. E.; DeGrado, W. F. Design, Synthesis and in vitro activities of a series of Benzimidazole/Benzoxazole Glycoprotein Ilb/IIla inhibitors. Bioorg. Med. Chem. Lett. 1996, 6, 339-344.

[9] Ramsbeck, D.; Buchholz, M.; Koch, B.; Böhme, L.; Hoffmann, T.; Demuth, H. U.; Heiser, U. Structure-Activity Relationships of Benzimidazole-Based Glutaminyl Cyclase Inhibitors Featuring a Heteroaryl Scaffold. J. Med. Chem. 2013, 56, 6613-6625.
[10] Arienti, K. L.; Brunmark, A.; Axe, F. U.; McClure, K.; Lee, A.; Blevitt, J.; Neff, D. K.; Huang, L.; Crawford, S.; Pandit, C. R.; Karlsson, L.; Breitenbucher, J. G. Checkpoint Kinase Inhibitors: SAR and Radioprotective Properties of a Series of 2-Arylbenzimidazoles. J. Med. Chem. 2005, 48, 1873-1885.

[11] Rollas, S.; Küçükgüzel, S. Biological Activities of Hydrazone Derivatives. Molecules. 2007, 12, 1910-1939.

[12] Kumar, V.; Basavarajaswamy, G.; Rai, M. V.; Poojary, B.; Pai, V. R.; Shruthi, N.; Bhat, M. Rapid 'one-pot' synthesis of a novel benzimidazole-5-carboxylate and its hydrazone derivatives as potential anti-inflammatory and antimicrobial agents. Bioorg. Med. Chem. Lett. 2015, 25, 1420-1426.

[13] Yadagiri, B.; Holagunda, U. D.; Bantu, R.; Nagarapu, L.; Guguloth, V.; Polepally, S.; Jain, N. Rational design, synthesis and anti-proliferative evaluation of novel benzosuberone tethered with hydrazide-hydrazones. Bioorg. Med. Chem. Lett. 2014, 24, 5041-5044.

[14] Şenkardes, S.; Kaushik-Basu, N.; Durmaz, İ.; Manvar, D.; Basu, A.; Atalay, R.; Küçükgüzel, Ş. G. Synthesis of novel diflunisal hydrazide-hydrazones as anti-hepatitis C virus agents and hepatocellular carcinoma inhibitors. Eur. J. Med. Chem. 2016, 108, 301-308.

[15] Cakir, B.; Dag, O.; Yildirim, E.; Erol, K.; Sahin, M. F. Synthesis and anticonvulsant activity of some hydrazones of 2-[(3H)-oxobenzoxazolin-3-yl-aceto]hydrazide. J. Fac. Pharm. Gazi. 2001, 18, 99-106.

[16] Siddiqui, A. I. S. M.; Macedo, T. S.; Moreira, D. R. M.; Leite, A. C. L.; Soares, M. B. P.; Azam, A. Design, synthesis and biological evaluation of 3-[4-(7-chloro-quinolin-4-yl)-piperazin1-yl]-propionic acid hydrazones as antiprotozoal agents. Eur. J. Med. Chem. 2014, 75, 67-76.

[17] Melnyk, P.; Leroux, V.; Sergheraert, C.; Grellier, P. Design, synthesis and in vitro antimalarial activity of an acylhydrazone library. Bioorg. Med. Chem. Lett. 2006, 16, 31-35.

[18] Garkani-Nejad, Z.; Ahmadi-Roudi, B. Modeling the antileishmanial activity screening of 5-nitro-2-heterocyclic benzylidene hydrazides using different chemometrics methods. Eur. J. Med. Chem. 2010, 45, 719-726.

[19] Siddiqui, S. M.; Salahuddin, A.; Azam, A. Synthesis, characterization and antiamoebic activity of some hydrazone and azole derivatives bearing pyridyl moiety as a promising heterocyclic scaffold. Eur. J. Med. Chem. 2012, 49, 411-416.

[20] Pieczonka, A. M.; Strzelczyk, A.; Sadowska, B.; Mlostoń, G.; Stączek, P. Synthesis and evaluation of antimicrobial activity of hydrazones derived from 3-oxido- $1 \mathrm{H}$-imidazole-4-carbohydrazides. Eur. J. Med. Chem. 2013, 64, 389-395.

[21] a) Hernandez, P.; Cabrera, M.; Lavaggi, M. L.; Celano, L.; Tiscornia, I.; Costa, T. R. D.; Thomson, L.; Fogolin, M. B.; Miranda, A. L. P.; Lima, L. M.; Barreiro, E. J.; Gonzalez, M.; Cerecetto, H. Discovery of new orally effective analgesic and anti-inflammatory hybrid furoxanyl $\mathrm{N}$-acylhydrazone derivatives. Bioorg. Med. Chem. 2012, 20, 2158-2171.

[22] Kakkerla, R.; Marri, S.; Krishna, M. P. S. M.; Molgara, P.; Reddy, Y. N. Synthesis and Biological Evaluation of 3,4-Dihydro-3-(3-methylisoxazol-5-yl)-2H-benzo[e][1,3] oxazine Derivatives as Anticancer Agents. Lett.Org. Chem. 2018, 15, 124-132.

[23] Kakkerla, R.; Marri, S.; Krishna, M. P. S. M.; Rajam, M. V. A facile and simple synthesis of novel isoxazolyl benzo[f][1,4] 
oxazepin-3-(2H)-ones and their antimicrobial activity. Indian J. Chem. 2018, 57B, 823-829.

[24] Marri, S.; Kakkerla, R.; Murali Krishna, M. P. S.; Venkat Rajam, M. Synthesis and antimicrobial evaluation of isoxazolesubstituted 1,3,4-oxadiazoles. Heterocycl. Commun. 2018, 24 , 285-292.

[25] Reddy, K. S.; Srinivasam, N.; Reddy, C. R.; Naveenkumar, K.; Anjaneyulu, Y.; Venkatraman, S.; Battacharya, A.; Mathad, V. T. An Efficient and Impurity-Free Process for Telmisartan: An Antihypertensive Drug. Organic Process Research \& Development. 2007, 11, 81-85.

[26] Chakrapani, L.; Raju, K. N.; Sridhar, K.; M. S. P. Sarma, M. P. S.; N. Choudary, N. WO Patent W02012/028925 A2, 2012.

[27] Kurumbail, R. G.; Stevens, A. M.; Gierse, J. K.; McDonald, J. J.; Stegeman, R. A.; Pak, J. Y.; Gildehaus, D.; Miyashiro, J. M.; Penning, T. D.; Seibert, K.; Isakson, P. C.; Stallings, W. C. Structural basis for selective inhibition of cyclooxygenase- 2 by anti-inflammatory agents. Nature, 1996, 384, 644-648.

[28] Matter, H.; Nazaré, M.; Güssregen, S.; Will, D. W.; Schreuder, H.; Bauer, A.; Urmann, M.; Ritter, K.; Wagner, M.; Wehner, V.
Evidence for $\mathrm{C}-\mathrm{Cl} / \mathrm{C}-\mathrm{Br}$...pi interactions as an important contribution to protein-ligand binding affinity. Angew. Chem. Int Ed. Engl. 2009, 48, 2911-2916.

[29] Rajasekaran, S.; Gopalkrishna, R.; Abhiskek, C. Synthesis, Anti-Inflammatory and Anti-oxidant activity of some substituted Benzimidazole Derivatives. Int. J. Drug. Dev. Res. 2012, 4, 303-309.

[30] Winter, C. A.; Risley, E. A.; Nuss, G. W. Carrageenin-induced edema in hind paw of the rat as an assay for anti-inflammatory drugs. Proc. Soc. Exp. Biol.1962, 111, 544-547.

[31] Tjølsen, A.; Rosland, J. H.; Berge, O. G.; Hole, K. The increasingtemperature hot-plate test: an improved test of nociception in mice and rats. J. Pharmacol. Meth. 1991, 25, 241-250.

[32] Zimet, P. O.; Wynn, R. L.; Ford, R. D.; Rudo, F. G. Effect of hot plate temperature on the antinociceptive activity of mixed opioid agonist-antagonist compounds. Drug Dev. Res.1986, 7, 277-280.

[33] Trott, O.; Olson, A. J. Aut dock Vina: improving the speed and accuracy of docking with a new scoring function, efficient optimization and multithreading. J. Comput. Chem. 2010, 31, 455-461. 